\title{
Participants Conform to \\ Humans but Not to \\ Humanoid Robots in an \\ English Past Tense \\ Formation Task
}

\author{
Clay Beckner', Péter Rácz', Jennifer Hay', \\ Jürgen Brandstetter', and Christoph Bartneck'
}

\begin{abstract}
In this article, we discuss the results of an experiment designed to test the boundaries of linguistic imitation in a group setting. While most prior work has focused on convergence in either sound structure or syntax, we investigate whether speaker's choices in verb morphology are influenced by others. The experiment is based on the peer pressure study of Asch. Participants give responses to target stimuli in a verbal and a visual task in a group of human peers, a group of robots, or alone. These results demonstrate that morphological conformity occurs, but that it is socially constrained-it happens with human peers but not with robot peers. This supports a view of linguistic convergence as a deeply social process. The level of linguistic conformity displayed by individuals is related to their degree of conformity in nonlinguistic tasks, suggesting that there are individual propensities toward peer imitation that transcend modalities.
\end{abstract}

\section{Keywords}

communication accommodation theory, morphological variation, lexical diversity, morphology, priming, conformity

Imitation between individuals is ubiquitous in human behavior, and its presence in linguistic interactions is well-attested (Giles, Coupland, \& Coupland, 1991; Soliz \& Giles, 2014). In a wide range of language domains, we find that speakers tend to converge on the same linguistic variant. An example comes from the work of Brennan and Clark (1996); when participants are asked to work together and describe images

'University of Canterbury, Christchurch, New Zealand

Corresponding Author:

Clay Beckner, New Zealand Institute of Language, Brain \& Behaviour, University of Canterbury, Private Bag 4800, Christchurch 8140, New Zealand.

Email: clayton.beckner@canterbury.ac.nz 
on cards to each other, they exhibit "lexical entrainment," by converging on shared reference terms amid a great range of possibilities (e.g., "pennyloafer"/"loafer"/ "shoe"/"leather shoe"/"docksider"). Participants in laboratory settings and natural conversations converge with respect to speech rate, accent, fundamental frequency, and choice of grammatical constructions (Bock, 1986; Estival, 1985; Giles, Taylor, \& Bourhis, 1973; Gregory \& Webster, 1996; Gries, 2005; Hall, Ferreira, \& Mayberry, 2015; Pardo, 2013; Webb, 1972). Mutual imitation in communicative settings extends beyond language proper, and includes such features as attunement of breathing rate (McFarland, 2001), smiling and foot-shaking (Chartrand \& Bargh, 1999). These imitative behaviors are associated with social variables, insofar as speakers shift their behaviors more toward interlocutors whom they regard favorably (Babel, 2012; Natale, 1975) or who have high prestige in the situation (Gregory \& Webster, 1996).

The present article investigates cross-speaker imitation in the domain of morphology, that is, the area of language that involves words and their meaningful subparts. Although there is extensive research on convergence in phonetics and syntax, work on imitative behaviors in morphology has been relatively scarce (noting exceptions in Szmrecsanyi, 2005, 2006). Morphological patterns can compete with one another, and certain English verbs have variable ways of marking the past tense (Haber, 1976; Moder, 1992). For example, the past tense of weave is variably observed to be a regular suffixed form (weaved), or an irregular form involving a vowel change (wove). The factors conditioning this variation have been discussed extensively (e.g., Rácz, Beckner, Hay, \& Pierrehumbert, 2014); however, we are not aware of any studies on convergence across such morphological patterns in the literature. Would hearing a speaker say weaved, for example, affect the likelihood that another speaker will opt for dived (rather than dove) as the past tense of dive?

If speakers indeed imitate one another's behaviors in morphology, the mechanisms at work are potentially multifaceted and worthy of investigation. A predominant view holds that convergence is primarily a cognitive phenomenon, as proposed, for example, by the interactive alignment model of Pickering and Garrod (Garrod \& Pickering, 2004; Pickering \& Garrod, 2004, 2006), which describes linguistic convergence as a mechanistic and automatic process. In the model, such processes arise from the priming of linguistic representations (at all levels), occurring in parity by the speakers as they interact. With particular respect to morphology, it is plausible that primarily cognitive processes are at work; note that morphological priming effects are regularly observed in the artificial environment of psycholinguistics labs, even from isolated words presented auditorily or onscreen (Crepaldi, Rastle, Coltheart, \& Nickels, 2010; Veríssimo \& Clahsen, 2009). Most relevantly to the current study, Moder (1992) presents a collection of irregular verb primes representing tokens of English irregular verb classes, such as sing-sang. During subsequent testing, participants' self-priming increases the likelihood that they will irregularize nonce forms that resemble the primed verbs in form. For instance, asking participants to provide the past tense of stride during the priming round makes it more likely that they will produce past forms that resemble strode when applicable (trive-trove, shrideshrode, and so on). Thus, it is conceivable that simply by virtue of repeated exposures 
to tokens representing a particular morphological pattern, speakers would converge unwittingly on shared verbal conjugations.

On the other hand, social factors may be primary in linguistic convergence. Communication accommodation theory (Gallois, Ogay, \& Giles, 2005; Giles et al., 1991; Soliz \& Giles, 2014) holds that convergence occurs because speakers seek social integration with one another, and as such, the degree of convergence will vary depending on the social situation, the interlocutors' perceptions of one another, and speakers' personality types. Even a principally cognitive model such as interactive alignment model makes room for social prerequisites regarding convergent processes, as explained by Pickering and Garrod (2004):

One can decide whether one is interacting with an agent with which it is appropriate to align. Thus, we can consider the interesting case of human-computer interaction, where people may or may not align with computers' utterances. If the conscious ascription of a mental state is necessary for alignment, then people will only align if they perform such ascriptions. But if people behave toward computers as "social agents", whatever they consciously believe about their mental states, then we predict unimpaired alignment will occur with computers, just as many other aspects of social behavior do [citing Reeves \& Nass, 1996]. (p. 188) [AQI]

These observations indicate that in investigating the mechanisms of convergence, it makes sense to consider the role of differing social reference points. In our study, we choose to include a nonhuman peer group as a boundary condition for the study of imitative behaviors. If peer influence is observed to occur equally with respect to humans and robots, then this would give evidence for the primacy of a cognitive priming mechanism in morphology, independent of social constraints. That is to say, we wish to control for the possibility that peer influence arises due to mere repetition of morphological targets. Repetition of -ed-final English past tense verbs, for instance, could have the effect of priming a linguistic unit (the -ed suffix) or priming a grammatical rule or pattern (forming the past tense by adding -ed), making the regularization of verbs more likely. In the present study, any differences between influence by humans and robots will provide evidence that the observed effect has a social component, and may provide insights about the social dynamics of human/robot interaction.

Our experiment has four aims: to determine whether conformity occurs in the domain of morphology, to compare linguistic conformity with conformity in a nonlinguistic task, to test for conformity on a general linguistic pattern as opposed to individual items, and to explore the social contexts in which conformity does and does not occur, by comparing the influence of human versus nonhuman peers. Our approach replicates and expands on landmark studies by Solomon Asch (Asch, 1951, 1955, 1956). In Asch's original methodology, participants are asked to perform a visual task in which they compare the lengths of different lines. Subjects are accompanied in the experimental room by several peers who answer each question first; however, the peers are confederates of the experimenter, and provide erroneous 
answers. In Asch's studies and a large number of replications (Bond \& Smith, 1996), participants are found to be influenced by the visual judgments of their peers. Our experiment represents the first extension of Asch's methodology tasks to the domain of language.

In this study, we are forging new connections between a classic experimental paradigm (Asch, 1951) and an extensive body of research on communicative accommodation. A few words on terminology and rationale are therefore appropriate. When referring to "convergence," we typically mean convergence in its most general sense, that is, to describe situations in which one speaker's linguistic forms increase in similarity to another speaker's utterances (cf. Pardo, 2013, on phonetic convergence). Yet we acknowledge that there are striking differences between most studies of convergence, as typically performed in communicative settings, and studies of conformity, as introduced by Asch (1951). First, convergent phenomena prototypically involve some kind of social interaction; for instance, Soliz and Giles (2014) define convergence as "a strategy whereby individuals adapt their communicative behaviors in such a way as to become more similar to their interlocutor's behavior" (p. 108). In contrast, an Asch-type conformity experiment incorporates no interlocutors per se; a participant hears responses by confederates, but these responses are not in themselves communicative.

Yet we should note that phonetic convergence has been observed not just in interactive experimental paradigms but also in noninteractive ones, such as studies in which subjects passively listen to recordings of target speech (Delvaux \& Soquet, 2007), or "shadow" recorded speech (Goldinger, 1998). Likewise, our conformity study presents target instances of language use, and examines whether participants match those targets. Moreover, a conformity paradigm has particular merit for studying the thresholds of social influence. Studies of human-machine interaction have found some evidence that speakers converge on the speech styles of their (computer or robot) interlocutors (for instance, Oviatt, Darves, \& Coulston, 2004). However, such results often have unclear implications about whether the speaker has regarded the interlocutor as a social peer. Speakers may adopt speech characteristics from the machine largely for purposes of communicative efficacy, that is, they may accommodate precisely because they have little confidence in the machine's abilities to understand (Branigan, Pickering, Pearson, \& McLean, 2010). In contrast, in the context of a peer pressure experiment, any observed shifts in participant behavior will necessarily have interesting implications regarding social mediation.

Second, we note that in conformity research, there are cases in which participants consciously override their own judgments in order to conform with the peer group. Asch (1956) reviews a number of posttask interviews with participants; in rare cases, subjects reported that they were unaware of having been influenced by the group. More often, however, participants noted that they were in some sense aware of having altered their answers, or having questioned their own perceptions, due to the answers of others; such experiences are in many ways different from real-world conversational interactions. 
On the other hand, we would argue that linguistic conformity (if it can be observed) has a great deal in common with linguistic convergence. If linguistic convergence is motivated by social factors, the underlying mechanisms undoubtedly overlap with the motivations for conformity in a peer pressure situation. Communication theorists have argued that speakers "intentionally and consciously make use of adaptive processes in order to ingratiate themselves to others" (Toma, 2014, p. 169). Many adaptations in communicative behavior are unquestionably unconscious (Chartrand \& Bargh, 1999), but others can be under conscious control, and socially variable-including, for instance, switching to a different pronunciation, slowing one's speech rate, or a switching from one language to another when speaking to a particular interlocutor. In communication accommodation theory, convergence is held to be motivated by a desire for social approval, or decreased social distance (Gallois et al., 2005; Soliz \& Giles, 2014). Such factors should be at play in a conformity experiment, as well as in conversational settings, whether or not individuals' social goals (and resulting decisions) enter conscious awareness. Having noted this common ground, when interpreting the present study we wish to draw on the insights of a large body of research on convergence in other linguistic domains, and to link the present experiment with a larger research project we have undertaken on social imitation in morphology.

\section{Study Variables and Hypotheses}

In order to see whether morphological conformity happens with any agent that the subject shares a task with, we recreated the Asch experiment with a group of human confederates as well as a group of humanoid robots. To see whether linguistic conformity is associated with nonlinguistic conformity, we included a visual and a verbal task. To find out whether abstract morphological patterns can constitute a basis for conformity, we used a setup in which participants respond to different items from confederates, and we then observe whether the target behavior (-ed past tense regularization) is generalized. We performed a $3 \times 2 \times 2 \times 2$ mixed-factor experiment. The within-subject variables were the modality of the stimulus (visual vs. verbal), and the ambiguity of the stimulus (ambiguous vs. nonambiguous). The visual task consisted of sets of lines, as in the design used by Asch. The verbal task consisted of lists of English verbs.

The between-subject variables were the ordering of the stimulus sets (visual task first vs. verbal task first); and the peer setting: a group of human peers, a group of robot peers, or no peers present (the baseline). The outcome variable was the number of times the responses of the participants conformed to the responses of their peers. In the visual task, the peer group provided incorrect visual judgments, and in the verbal task, the peer group always provided the regular (-ed-final) past tense form. We cannot talk of conforming to peers in the baseline setting, where participants were alone. In this setting, the outcome variable was an incorrect response in the visual task and the selection of the regular past in the verbal task. The main variables of the experiment can be seen in Table 1 . 
Table I. Variables of the Experiment.

\begin{tabular}{lllll}
\hline & Peer setting & Ordering & Modality & Ambiguity \\
\hline $\begin{array}{l}\text { Within- or between-subjects } \\
\text { Variables }\end{array}$ & $\begin{array}{l}\text { between } \\
\text { baseline } \\
\text { robot peers } \\
\text { human peers }\end{array}$ & $\begin{array}{l}\text { between } \\
\text { visual first }\end{array}$ & $\begin{array}{l}\text { within } \\
\text { visual within } \\
\text { ambiguous }\end{array}$ \\
& & verbal nonambiguous \\
\end{tabular}

We have four hypotheses, as follows.

1. In both the visual and the verbal task, human confederates will induce conformity. Based on related findings in linguistic convergence, we predict that participants will imitate the morphological judgments of human peers. We predict that robots should prompt some imitation as well. As noted by Pickering and Garrod (2004), there is evidence that people treat computers (and robots) as social peers, for instance, when respecting politeness norms while levying criticism at machines (Nass, Moon, \& Carney, 1999; Nass, Steuer, \& Tauber, 1994; Reeves \& Nass, 1996). Based on the assumption that robots are borderline social agents, it is predicted that they will have some influence on the responses of participants in the verbal and visual tasks. However, conformity with robots peers will be markedly diminished compared with human peers.

2. Conformity will be higher for ambiguous items than for non-ambiguous items, and we predict this will hold true for both the verbal and the visual task. With respect to the verbal task, the ambiguous verbs are, by definition, items on which there is attested variation between regular and irregular pasts (weaved and wove are both acceptable). Thus for these items, -ed regularization of the form lies within the normal bounds of speech behavior, and participants may be especially inclined to regularize them when peers exhibit similar behavior on related items.

3. An individual participant's degree of conformity in the visual task will be related to their degree of conformity in the verbal task. Prior research on conformity has been largely confined to the same visual judgment task investigated by Asch (1951; though see Kundu \& Cummins, 2013, on conformity in moral judgments). As we extend the methodology to a new domain, it is natural to investigate whether effects are specific to the domain in which imitation occurs, or perhaps resultant from individual personality characteristics. We thus examine conformity in a visual task alongside a verbal task, and predict that social influenceability is an individual difference across participants. Some individuals will attend more closely to the judgments of peers than others, and this individual propensity should hold true across domains.

4. In the verbal task, conformity will be observed on the first verb a participant produces in a stimulus round, on the basis of peers' regularization of related (but different) items. That is, participants will be inclined not just to regularize 
items that their peers have already regularized, but they will also be prone to regularize new items, on the basis of overall behavior on similar items by peers. We thus seek evidence that participants will exhibit a combination of mimicry (insofar as they copy a pattern) and novelty (insofar as they extend patterns to new items). The ability to extend patterns to novel instances is a hallmark of verbal productivity (Moder, 1992) and is a likely mechanism in language change (Bybee, 1985; Bybee, 2010).

\section{Method}

Design

Presentation of Stimuli. In the visual decision task, each target stimulus consists of three parallel lines labeled A, B, C on the left side of the projection, and one line labeled with ? on the right side. The line on the right, marked with a ?, matches one of the lines on the left. The task of the participant was to say which line, A, B, or C, matches the line labeled?. Target stimuli were presented on a large screen using a projector.

In the spoken task, each target stimulus consists of a group of five verbs. The task was to read a given verb out loud, and then provide its past tense form, for example like and liked. Each respondent had to say the verb corresponding to their position in the peer group. This means that respondent one would say the first verb and its past tense, respondent two would say the second verb and its past tense, and so on. The order of the respondent was always the same, such that the four confederates answered first, and the experimental participant answered last. An example stimulus round is shown in Figure 1, illustrating (a reenactment of) the peer group setting for a human participant and robot confederates.

Baseline Studies. We ran two baseline studies to provide reference points for judgments in the visual and verbal tasks, in separate groups of participants who performed the tasks alone, and who thus had no pressure from peers.

For the visual task, we collected data that functioned both as a pilot study - since it identified easy and difficult visual judgments for further investigation-and as a statistical baseline for the peer group settings. The full pilot set consisted of 107 line configurations. The setup of the room was exactly the same as in the group experiment, the only difference being that the participants did not say out loud which line best matched; they used the buttons 1, 2, 3 on the keyboard. We assume there is no difference between saying the answer aloud or pressing a button when no one is in the room other than the participant. Out of these 107 settings, we picked 30 for our group experiment, divided into two parts. The first part (15 settings) selected the 15 line settings which prompted the most errors across participants. These line comparisons were not easy to resolve, and were thus considered the "ambiguous" lines in the peer conditions. The second part ( 15 settings) were selected randomly from all line length comparisons that differed by $30 \%$ or less, following parameters chosen by 


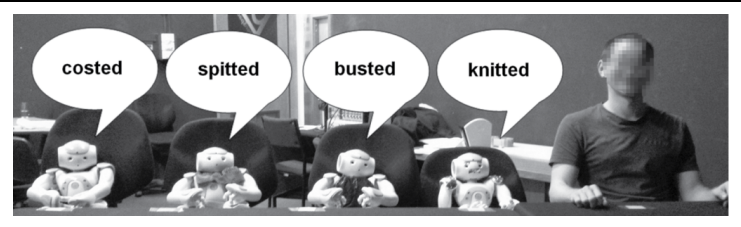

Figure I. An example of a stimulus round presented in the verbal task.

Note. Onscreen, the verbs cost, spit, bust, knit, and wet appear. The four confederates first respond with the regular past tense form of the first four verbs, as illustrated here for the robot peer condition. The participant must then provide the past tense form of the fifth verb, wet.

Asch (1951) to prevent the task from being too easy. It was verified that participants in the pilot made only one or fewer mistakes on these items, and they were designated as "non-ambiguous" stimuli. The errors on these 30 targets were then used as the baseline comparison for errors by participants in the robot and human peer conditions.

The baseline condition for the verbal task was the same as the peer conditions, except that participants had to produce past tenses for all the forms on the screen. All items that were presented in the peer conditions (to confederates as well as participants) were included in the baseline, to control for possible priming effects. However, for purposes of quantitative comparison between baseline and peer conditions, we only analyze the 30 verbs for which participants in the peer groups also gave responses. We used consistent coding for the baseline and the group settings, labeling only regularized responses as "conforming." Unlike the peer group settings, the baseline settings for the lines and the words were run with two different sets of participants. ${ }^{1}$

Groupings of Stimuli. Each experimental block (involving either lines or verbs) was divided into four parts: an example (1 target), a warm-up (3 targets), an ambiguousitem set (15 targets), and a nonambiguous-item set (15 targets). Each experiment block thus consisted of 34 targets. Warm-up and example targets are not analyzed, leaving 30 responses per subject in each block.

The warm-up consisted of three unambiguous tasks. It had relevance for the peer group settings. The peer group would say the correct answer-thus providing the correct visual judgment in the visual task, or providing the normative/common past tense in the verbal task. This was to convince participants that the robots/actors understood the question, and could provide correct responses.

In the third and fourth parts of each experiment block, the confederates (robots or human actors) gave consistent answers, with the intention of swaying participants' responses. In the visual task, the confederate actors/robots would always choose the 
incorrect line response. In the verbal task, the confederates always provided the regular past tense form, that is, a form which ends in -ed.

In the verbal task, our design is such that on each round, participants and confederates respond to different items, and thus, the influence from one item to another requires generalization of a pattern, rather than mere repetition of particular items. Ambiguous verb stimuli are those for which variation exists in English usage, and the target words can have a regular or irregular past tense form, for example, divedived, or dive-dove. Nonambiguous verbal stimuli are items that have only an irregular past tense form, for example, run-ran. Note that this means that in the peergroup conditions, the robots/actors all provided verb forms that are not normatively in use, that is, overregularized forms such as runned as the past tense of run. Appropriate candidates for ambiguous and nonambiguous items were identified by searching the CELEX Lexical Database for English verbs that do or do not exhibit variation (Baayen, Piepenbrock, \& Gulikers, 1995).

The ambiguous verbal stimuli are organized on the basis of verb groups, that is, sets of items which resemble one another in the form of their past tense alternation. Such groupings arise from historical English verb classes, and give signs of being interconnected in mental representations (Bybee, 1985; Bybee \& Slobin, 1982; Stemberger \& MacWhinney, 1986). The five groupings used for our ambiguous verbal stimuli are as follows:

- SHRANK. Verbs that form an irregular past with a vowel change from [I] to [æ] (e.g., ring-rang, sink-sank, shrink-shrank).

- LEARNT. Verbs that form an irregular past tense by adding a [t], with no change in the stem vowel (e.g., learn-learnt, spill-spilt, spoil-spoilt). These items constitute a distinct set from regular English pasts such as boss-bossed which are articulated with a $[\mathrm{t}]$ allomorph, insofar as the learnt verb bases actually end in a voiced consonant but are nonetheless affixed with a voiceless stop.

- DREAMT. Verbs that form an irregular past by changing the stem vowel from [i] to [E] (e.g., dream-dreamt, plead-pled, kneel-knelt).

- DOVE. Verbs that form an irregular past with a vowel change from [a I] or [i] to [ou] (e.g., drive-drove, weave-wove, ride-rode).

- KNIT. No-change past tense verbs, that is, verbs that have an irregular past tense which is identical to their present tense form (e.g., knit-knit, cost-cost, wet-wet). Verb bases in this class end in sounds that are already associated with the English past tense, that is, [t] or [d] (Bybee \& Slobin, 1982), although the nonce verb bases in the present study all end in $[\mathrm{t}]$.

The literature contains various taxonomies of English irregular verb classes, but our current classification mostly (if not entirely) represents a subset of the detailed verb classes outlined by Moder (1992).

Each presentation round during the ambiguous verbal portion of the experiment consisted of five ambiguous verbs drawn from the same verb group. The 
nonambiguous verbs were not organized into phonological groups, but they were also presented in groups of five. The list of ambiguous and nonambiguous verbs can be found in the appendix. We randomized the order of verb groups within the ambiguous and nonambiguous blocks, and also randomized the order in which the verbs occurred in each group. Individual random presentation orders were created for individual participants within a peer-group condition, although randomizations were reused across conditions (robot peer setting, human peer setting, and baseline setting) to match as many details as possible. Multiple presentations of the same verb group occurred (with different orderings, and different response items on each presentation). Participants only responded to three targets from each group of five verbs so as to keep the experiment under 30 minutes.

In the peer group settings, all participants performed both the verbal and visual tasks. We counterbalanced the order of the verbal and visual experiment blocks.

\section{Apparatus}

The setting consisted of a projector, a high-quality wireless microphone, a table with five chairs, four customized Aldebaran NAO robots or four confederate actors, and a laptop to control the recordings and the experiment. The projecting area had a dimension of $243 \times 177 \mathrm{~cm}$, although the maximum line length was $118 \mathrm{~cm}$. The table was exactly parallel to the projecting area at a distance of $200 \mathrm{~cm}$. The dimensions of the table were $80 \times 250 \mathrm{~cm}$

To make our NAO robots more individualistic, we gave each robot a different outfit (as shown in Figure 1) and voice. We did this individualization process on the assumption that if robots were distinctive, participants would more easily see them as individuals rather than as copies of each other. For the robot voices, we used recordings of the same actors who assisted us in the human peer condition. We also made sure that each robot (in the robot condition) sat in the position corresponding to the actor (in the human condition) whose voice it shared.

\section{Procedure}

The experiment was designed to discourage spontaneous interactions between the participant and the confederates. In both conditions, the participant signed consent forms in a room separate from the experiment room. The participant was then informed that the other participants were already in the experiment room and the study was ready to begin. When the participant entered the second room, all the confederates greeted him or her (with a simple "Hello," "Hi there," etc.). In the robot condition, the robot confederates turned their heads toward the participant when making the greeting, to give a sense that the robots were aware of the participant's presence. The research assistant told the participant to find an empty chair; since the confederates were already seated, the participant had no option but to take the last chair in the row. After the participant sat down, the experiment immediately started. 
We used prerecorded instructions to participants in all conditions. The experiment lasted between 20 and 30 minutes.

\section{Participants}

We had 78 participants; 23 in a peer group with human confederates, 19 in a peer group with robots, 18 in the visual task baseline, and 18 in the verbal task baseline. All participants were college students at the University of Canterbury, and all were native speakers of New Zealand English.

No participants reported any previous familiarity with the Asch experiment during debriefing. All participants were paid $\$ 10$ for their participation.

Participants' responses were recorded digitally and transcribed by a native speaker of New Zealand English.

\section{Results}

Figure 2 shows the percentages of responses grouped according to setting, modality, and ambiguity. Responses with the human peer group have high conformity, whereas responses with the robot peer group are similar to baseline responses. For the nonambiguous verbs, participants in isolation regularized on $6 \%$ of responses, compared with $11 \%$ for those with robot peers and $27 \%$ of those with human peers. For the ambiguous verbs, $39 \%$ of responses by baseline participants regularized the verb, compared with $43 \%$ for robot-peer participants and $59 \%$ for human-peer participants. In both these cases, the robot condition tends in the expected direction, although (as demonstrated in the statistical models below), there was no significant difference from baseline. In contrast, the participants with human peers were significantly different from both baseline and robot conditions. The pattern is similar in the visual task - although here, the robots did not tend in the expected direction for the nonambiguous items, with a (nonsignificant) decrease in error rate from $2 \%$ to $1 \%$.

We fit a logistic mixed-effects regression model on the full data set, using stepwise regression and maximal random effects structure (Breslow \& Clayton, 1993; Jaeger, 2008). Model comparison proceeded such that all independent variables and interactions, as listed in Table 1 , are included initially, and dimensions that are not significant are dropped in stepwise fashion from the model (Barr, Levy, Scheepers, \& Tily, 2013). The outcome variable is whether the response matches the majority answer in the peer group conditions, that is, incorrect answers for the visual task, and regular -ed past tenses in the verbal task. The predictors are setting (baseline/robots/humans), ordering (visual task first/verbal task first), modality (visual task verbal task), and ambiguity (ambiguous/nonambiguous).

The regression model summary is shown in Table 2 . The results indicate that responses in the human peer group differ significantly from baseline responses ( $p<$ $.0001)$, whereas responses in the robot condition did not. Participants are more likely to match their peers on ambiguous stimuli than nonambiguous stimuli $(p<.0001)$. 


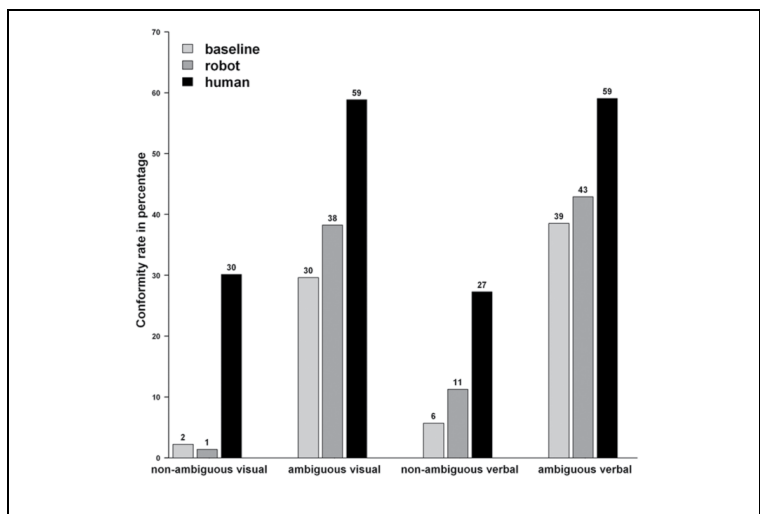

Figure 2. Percentage rate of conformity: All items and conditions.

Note. In the baseline condition, "conformity" represents the behavior of interest (error in the visual task, and regularization in the verbal task) by subjects who had no peer influence, and is thus used as a reference point for matched items in the peer conditions.

Table 2. Mixed Effects Model Summary: All Items, in Visual and Verbal Tasks.

\begin{tabular}{lcccc}
\hline \multicolumn{4}{c}{ Conformity $\sim$ peer setting $\times$ ambiguous $+(I+$ ambiguous } & subject $)+(I+$ peer setting $\mid$ target $)$ \\
\hline (Intercept) & Coefficient & SE & $\mathrm{z}$ & $p$ \\
Peer setting: Humans & -5.53 & 0.57 & -9.79 & $<.0001$ \\
Peer setting: Robots & 3.88 & 0.72 & 5.40 & $<.0001$ \\
Ambiguous: TRUE & 0.59 & 0.84 & 0.69 & .49 \\
Humans $\times$ Ambiguous & 4.80 & 0.55 & 8.66 & $<.0001$ \\
Robots $\times$ Ambiguous & -2.53 & 0.61 & -4.16 & $<.0001$ \\
& -0.27 & 0.74 & -0.37 & .71 \\
\hline
\end{tabular}

This is significantly stronger within the human peer group; indeed, there is a positive interaction between ambiguous stimuli and the presence of human peers $(p<.0001){ }^{2}$ Ordering and modality are not significant predictors of responses, and thus are not included in the model summary.

As seen in Figure 3, responses vary according to verb group within the ambiguous verb set. We fit a regression model on the verb responses only, including verb group as a predictor. The model summary can be seen in Table 3 . There is no interaction between peer setting and verb group, that is, the rate of conformity depends on the setting (human peers versus other, $p=.003$ ) and on the verb group but not on both 


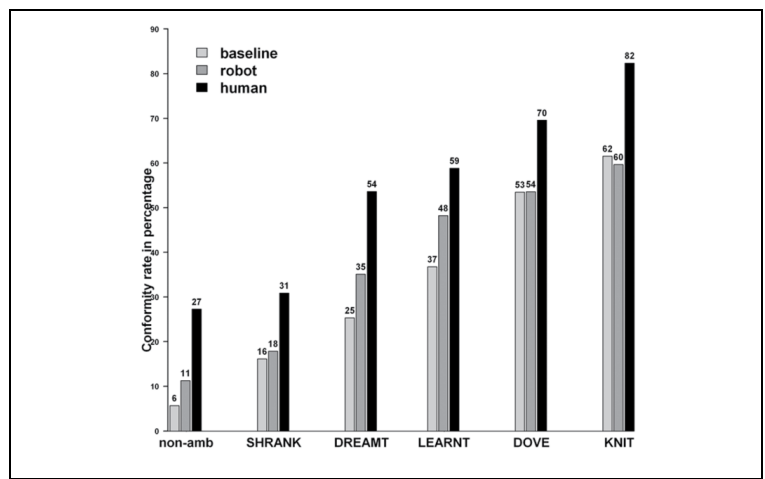

Figure 3. Percentage rate of conformity: Verbs only, by group.

Note. As before, in the baseline condition, "conformity" represents the behavior of interest (error in the visual task, and regularization in the verbal task) by subjects who had no peer influence, and thus used as a reference for matched items in the peer conditions.

Table 3. Mixed Effects Model Summary: Ambiguous Verbs Only, by Verb Group.

\begin{tabular}{|c|c|c|c|c|}
\hline \multicolumn{5}{|c|}{ Conformity $\sim$ peer setting + verb group $+(\mathrm{I} \mid$ subject $)+(\mathrm{I} \mid$ target $)$} \\
\hline & Coefficient & SE & $z$ & $p$ \\
\hline (Intercept) & -2.19 & 0.94 & -2.34 & .02 \\
\hline Peer setting: Humans & 1.36 & 0.46 & 2.97 & .003 \\
\hline Peer setting: Robots & 0.20 & 0.48 & 0.41 & .68 \\
\hline Verb group: KNIT & 3.10 & 1.24 & 2.49 & .013 \\
\hline Verb group: LEARNT & 1.58 & 1.24 & 1.27 & .20 \\
\hline Verb group: DOVE & 2.79 & 1.25 & 2.23 & .02 \\
\hline Verb group: DREAMT & 1.00 & 1.24 & 0.81 & .42 \\
\hline
\end{tabular}

together. The order in which the verb groups induce regularization (which is the conforming response), namely, SHRANK $<$ DREAMT $<$ LEARNT $<$ DOVE $<$ KNIT, is strikingly similar to the ratings observed in an online regularization task using nonce verbs (Rácz et al., 2014). This further supports the validity of this model.

We proposed that responses in the visual task would be good predictors of responses in the verbal task but only in a peer group that induces conformity. That is, participants who are more sensitive to peer pressure - as shown by their conformity ratings in the visual task-will show more linguistic conformity in a social context which elicits such conformity. 
Table 4. Mixed Effects Model Summary: Using Conformity in the Visual Task (Line Judgments) as a Predictor for Conformity in the Verbal Task (Regular Past Tense) (Peer Group Settings Only).

\begin{tabular}{|c|c|c|c|c|}
\hline \multicolumn{5}{|c|}{$\begin{array}{c}\text { Verbal task conformity } \sim \text { peer setting } \times \text { ambiguous }+ \text { peer setting } \times \text { visual task conformity } \\
+(\mid+ \text { ambiguous } \mid \text { subject })+(\mid+ \text { peer setting } \mid \text { target })\end{array}$} \\
\hline & Coefficient & $S E$ & $z$ & $p$ \\
\hline (Intercept) & -1.95 & 0.85 & -2.31 & .02 \\
\hline Peer setting: Robots & -4.00 & 1.25 & -3.19 & .001 \\
\hline Ambiguous: TRUE & 2.84 & 0.81 & 3.52 & $<.001$ \\
\hline Visual task conformity & 1.13 & 0.35 & 3.27 & .001 \\
\hline Robots $\times$ Ambiguous & 2.74 & 1.01 & 2.72 & .006 \\
\hline Robots $\times$ Visual task conformity & -1.55 & 0.51 & -3.03 & .002 \\
\hline
\end{tabular}

To investigate this hypothesis, we fit a separate regression model on the verbal responses in the peer groups only (excluding the baseline), and used the participants' mean conformity rating in the visual task as an additional predictor. The model summary can be seen in Table 4.

Although conformity with the peer group on visual judgments is a significant predictor of verbal responses for both robot and human peer groups $(p=.002)$, its effect is much larger in the human group. A setting: Visual conformity interaction analysis of the model in Table 4 yields an effect size of .55 for the human peer group, as compared with .004 for the robot group; a plot of this interaction is presented in Figure 4.

Finally, consider the role of cross-item generalizations among the experimental stimuli. Recall that the verbal targets were organized into groups of five based on morpho-phonological patterning. In the robot and human conditions, there were five respondents to each verb group presentation, the last being the participant. Due to time constraints, each verb group was presented three times. This means that there was a first time when the participant gave a past tense response to a particular verb without having heard that same verb from any of their peers. The second and third response to the verb group (with different verbs as targets) already followed a response for the specific target verb by one of the peers earlier in the experiment (once and twice, respectively). These responses could therefore be interpreted as repetitions, primed by the target itself. In contrast, the first response can only be primed by the overall pattern of behavior and not the particular item.

To test for non-token-based priming, we fit a regression model on first mentions of a target only. The model summary can be seen in Table 5. Setting remains a significant predictor (for human peers, $p=.015$ ), as does ambiguity $(p<.001)$. People conform more to human peers than to robots, and there is no significant difference between responses in the robot peer and baseline conditions.

This is the first time morphological imitation between speakers has been observed in a laboratory setting. In their conjugation of previously unheard items, subjects in our human-peer condition are swayed to a significant degree by the overall morphological pattern exhibited by other speakers. As in Moder (1992), we find that a 


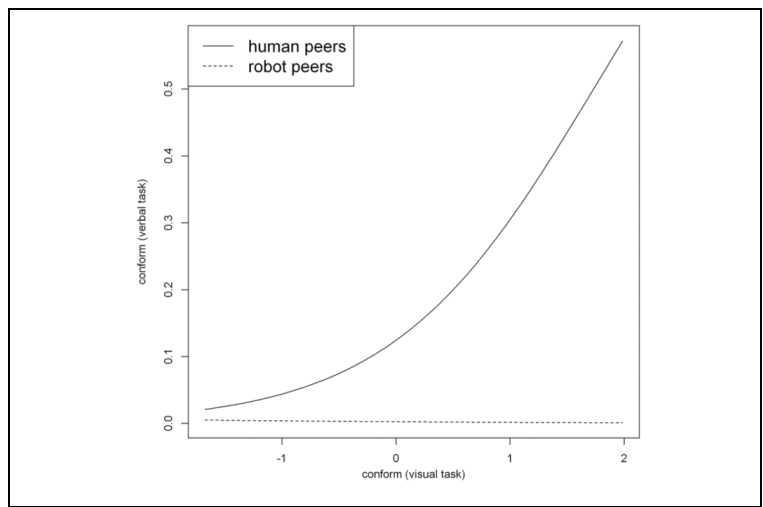

Figure 4. Interaction plot from the model in Table 4, for Peer setting $\times$ Visual task conformity.

Table 5. Mixed Effects Model Summary: First Mentions of Verbs (Verbal Stimuli Only, First Mentions Only).

\begin{tabular}{lcccc}
\hline \multicolumn{5}{c}{ Verbal conformity $\sim$ peer setting $\times$ ambiguous $+(I+$ ambiguous $\mid$ subject $)+$} \\
& $(I+$ peer setting & target $)$ & & \\
\hline & Coefficient & $S E$ & $z$ & $p$ \\
\hline (Intercept) & -5.40 & 1.03 & -5.24 & $<.000 \mathrm{I}$ \\
Peer setting: Humans & 3.12 & 1.18 & 2.64 & .008 \\
Peer setting: Robots & -0.01 & 1.50 & -0.01 & .99 \\
Ambiguous: TRUE & 5.31 & 1.03 & 5.15 & $<.0001$ \\
Humans $\times$ Ambiguous & -1.94 & 1.05 & -1.85 & .064 \\
Robots $\times$ Ambiguous & 0.27 & 1.39 & 0.19 & .85 \\
\hline
\end{tabular}

primed morphological pattern spreads from one item to other items. However, in the present experiment it is evident that the priming effect is socially constrained, since it arises only when relevant tokens are provided by human peers, as opposed to robots.

We note further that first mentions, in general, have a higher conformity rate than subsequent mentions. This is, to some extent, an artefact of the experiment design. Conformity decreased continuously during the experiment, as participants (1) encountered items that were less likely to be conjugated regularly, since nonambiguous items occurred as a block after ambiguous items and (2) (likely) became more and more suspicious of the peer group. Since first mentions came first, 
Table 6. Significant Variables of the Experiment, Emphasized With Asterisks.

\begin{tabular}{lllll}
\hline & Peer setting* & Ordering & Modalitya & Ambiguity* \\
\hline Within- or between-subjects & between & between & within & within \\
Variables & $\begin{array}{l}\text { baseline } \\
\text { robot peers } \\
\text { human peers* }\end{array}$ & visual first & visual & ambiguous* \\
& & &
\end{tabular}

a Task modality is not a significant predictor in our regression models, that is, conformity on the visual task is not distinguished from conformity on the verbal task. Follow-up analyses show that visual task conformity* is a significant predictor of verbal task conformity (see Table 4).

they always had higher conformity ratings than the corresponding later responses. Curiously enough, this pattern does not seem to hold across modalities, since the order of verbal and visual tasks was not a significant predictor of conformity with the peer group.

\section{Discussion}

Our study helps to shed new light on the bounds of linguistic convergence- by delineating a social threshold necessary for linguistic peer influence, and by demonstrating that subjects are influenced by the morphological choices of their peers. Our findings suggest that imitative behavior in morphology is a socially mediated behavior, in which the social situation, the individual's overall propensity to converge, and the degree of natural malleability of the stimulus all play a role. We repeat the variables of our experiment in Table 6, emphasizing the significant ones.

Our first hypothesis was that human peers would prompt conformity in the experiment, and that conformity with human peers would be greater than that with robots. Indeed, the data indicate that participants conform to human peers in both the verbal and visual tasks, thus providing evidence that imitative behavior occurs in morphology, as in other linguistic domains. Future work will expand on this finding, and investigate morphological convergence in settings that incorporate more interaction between interlocutors.

We also predicted that some conformity to robot peers would occur, but the evidence does not support this prediction. The behavior of participants exposed to judgments by robot peers was not significantly different from behavior by isolated participants (the baseline). This null finding, of course, need not challenge the claim that robots may be regarded as "social actors" in some contexts (Reeves \& Nass, 1996), though the data suggest that people failed to ascribe robots some crucial element in our experiment - perhaps a capacity for social pressure (arising from a presumed theory of mind). The corresponding implications about human interaction are instructive: It is apparent that participants are swayed by the behaviors of others, simply by virtue of their status as humans. People have a tendency to imitate one another in linguistic and nonlinguistic domains, even when the parties are strangers, when the task involves no interaction between parties, and when nonimitation carries no apparent consequences. 
Robots are not given the benefit of the doubt in these capacities. It remains possible that robots could "earn" peer status with appropriate measures, for instance, by demonstrating more variable behaviors, by demonstrating linguistic sophistication prior to the task, or by engaging the participant in social chitchat. These areas remain to be investigated in our future research.

We note that prior studies of speaker accommodation to machines (Branigan et al., 2010) generally require the participant to communicate with the computer, and it is likely that communicative interactions are important for prompting linguistic shifts toward nonhuman peers. Conversely, we should reiterate that, as observed by Branigan et al. (2010), linguistic alignment with machines may be principally motivated by communicative efficacy (that is, participants' supposition that machines have limited language skills) rather than true social accommodation. Studies reporting accommodation to computers and robots should thus be evaluated carefully to understand the mechanisms at work. In the current experiment, we have addressed this issue by incorporating a decidedly non-interactive platform for peer influence. Interestingly, this approach suggests that humans influence one another even in this artificial context (replicating Asch, 1951), but that robots do not produce the same effect.

Our second hypothesis was that the ambiguity of stimuli would have an effect on degree of conformity. As predicted, conformity is larger for the ambiguous items than for the non-ambiguous ones, and moreover, the presence of human peers interacts with the ambiguity of the stimulus. These results are consistent with the phenomenon of "social proof": In uncertain situations, humans are particularly attentive to and susceptible to the influence of other people (Sherif, 1935).

However, we also find that human peers are able to exert enough pressure to cause a significant amount of conformity on non-ambiguous items as well. The co-opting of unexpected, non-ambiguous innovations is a particularly striking finding with respect to the linguistic task, insofar as participants volunteered significantly more ungrammatical answers such as runned and goed. Such behavior arose imitatively, prompted when the participants had human peers, but not when the peers were robots.

Our third hypothesis was that we could observe meaningful proclivities in individual participants, when comparing behaviors across the verbal and visual tasks. The results indeed indicate that linguistic conformity is similar to social accommodation in a visual decision task; we have not found differences between the response patterns for the visual and the verb modalities. While the two are not trivial to compare (these being qualitatively different tasks in their experimental setup), it is also suggestive that we found a significant difference between baseline and human peer group responses for both tasks, and no such difference between baseline and robot peer group for either task. The former result also validates the latter one inasmuch as it shows that the experimental design works - at least with human peers. A further piece of evidence for the similarity of participant behavior in the two types of tasks is that participants who have a higher conformity rating on the visual task also have a higher conformity rating on the verbal task. This finding is true for both peer conditions, although the effect is much weaker in the robot peer condition than in the 
human peer condition. The overall data set points toward a domain-general account of conformity, including social influenceability in linguistic as well as nonlinguistic behaviors.

Finally, we hypothesized that in the verbal task, generalizations across items can be observed, as demonstrated most clearly by analyzing the responses on first mentions of a given verb target. Since the subject and confederates responded to different verbal items on each round, on first mentions subjects were responding to items they had not yet heard produced by their peers. In the human confederate condition, subjects nevertheless converged on the same morphological pattern as their social peers. These results can illuminate the ways linguistic innovations spread in the speaker community; one speaker's utterance of one lexical item can activate a range of related items. Imitation - paired with novelty - is implicated in processes of language change, as speakers adopt new speech variants from each other (Babel, 2012; Brennan \& Clark, 1996; Pardo, Gibbons, Suppes, \& Krauss, 2012; Sancier \& Fowler, 1997), and extend existing patterns to new contexts (Labov, 2007). Our study supports a growing body of research finding that social factors are important in processes of speech processing, imitation, and ultimately, language change (Baxter, Blythe, Croft, \& McKane, 2009; Hay, Warren, \& Drager, 2006; Hruschka et al., 2009). The verbal responses in our experiment reveal that participants imitate one another's morphological patterns - but only under the influence of peers who pass social benchmarks, that is, status as humans.

\section{Appendix}

List of Verb Stimuli.

\begin{tabular}{lll}
\hline & Ambiguous stimuli & Nonambiguous stimuli \\
\hline KNIT & knit & say \\
& cost & take \\
& spit & find \\
& bust & hear \\
DOVE & wet & stand \\
& dive & make \\
& heave & think \\
& drive & leave \\
& weave & bring \\
LEARNT & thrive & lose \\
& spill & come \\
& smell & know \\
& spoil & feel \\
& spell & sit \\
DREAMT & learn & keep \\
& plead & go \\
& kneel & tell \\
& leap & put \\
& dream & hit \\
SHRANK & lean & read \\
& sing & get \\
\hline
\end{tabular}




\begin{tabular}{ll}
\hline sink & see \\
shrink & give \\
ring & hold \\
spring & write \\
\hline
\end{tabular}

Note. Verb stimuli used in the experiment. Stimuli are listed here in the clusters of five items that constituted presentation rounds. For the ambiguous stimuli, these sets are labeled with the name of the verb group.

\section{Acknowledgments}

We are grateful for research help from Latifah Almanea, Natalie Eustace, Julian Melchert, Morgana Mountfort-Davies, Jacqueline Nokes, Timothy Pomroy, Eduardo Sandoval, Elizabeth Youard, Patrick LaShell, and Janet Pierrehumbert. We also wish to thank Howard Giles and two anonymous reviewers for helpful feedback on earlier drafts of this article. This project was made possible through the support of a subaward under a grant to Northwestern University from the John Templeton Foundation. Hay and Beckner were also supported by a Rutherford Discovery Fellowship awarded to Hay. The opinions expressed in this publication are those of the author(s) and do not necessarily reflect the views of the John Templeton Foundation or the Rutherford Foundation.

\section{Declaration of Conflicting Interests}

The author(s) declared no potential conflicts of interest with respect to the research, authorship, and/or publication of this article.

\section{Funding}

The author(s) disclosed receipt of the following financial support for the research, authorship, and/or publication of this article: This research was supported by the John Templeton Foundation (Award ID 36617) and the Rutherford Foundation (Grant Number E5909).

\section{Notes}

1. The use of two different subject groups for the baseline studies is not an essential feature of the experiment design but an artefact of earlier stages of this study. During analysis, it came to our attention that it would be necessary to gather verbal baseline data, rather than relying on corpus data to observe verbal conjugations in the absence of peer influence. The use of distinct baseline populations only has relevance with respect to our findings that compare the visual task with the verbal task. Our observations about cross-task generalizations would be questionable only in the unlikely event that there is an inherent relationship between our tasks (minus peer influence), that is, if people who tend to make many visual errors are also particularly prone to using the English -ed past tense. In any event, statistical models which separately analyze the verbal responses and the visual responses reach conclusions identical to the combined models presented here.

2. In interpreting the direction of effects and interactions in Table 2, note that the regression coefficients must be compared against the coefficient of the model intercept, that is, the baseline setting. Where possible, our regression models generally establish the baseline setting as the intercept, since this approach provides the most intuitive interpretation of the 
behavior of subjects in peer groups, as compared with behavior on matched items by isolated participants.

\section{References}

Asch, S. E. (1951). Effects of group pressure upon the modification and distortion of judgments. In H. Guetzhow (Ed.), Groups, leadership, and men (pp. 177-190). Pittsburgh, PA: Carnegie Press.

Asch, S. E. (1955). Opinions and social pressure. Scientific American, 193, 31-35.

Asch, S. E. (1956). Studies of independence and conformity: I. A minority of one against a unanimous majority. Psychological Monographs: General and Applied, 70, 1-70.

Baayen, R. H., Piepenbrock, R., \& Gulikers, L. (1995). The CELEX lexical database (CDROM). University of Pennsylvania, PA: Linguistic Data Consortium.

Babel, M. (2012). Evidence for phonetic and social selectivity in spontaneous phonetic imitation. Journal of Phonetics, 40, 177-189.

Barr, D. J., Levy, R., Scheepers, C., \& Tily, H. J. (2013). Random effects structure for confirmatory hypothesis testing: Keep it maximal. Journal of Memory and Language, 68, 255-278.

Baxter, G. J., Blythe, R. A., Croft, W., \& McKane, A. J. (2009). Modeling language change: An evaluation of Trudgill's theory of the emergence of New Zealand English. Language Variation and Change, 21, 257-296.

Bock, J. K. (1986). Syntactic persistence in language production. Cognitive Psychology, 18, 355-387.

Bond, R., \& Smith, P. B. (1996). Culture and conformity: A meta-analysis of studies using Asch's (1952b, 1956) line judgment task. Psychological Bulletin, 119, 111-137.

Branigan, H. P., Pickering, M. J., Pearson, J., \& McLean, J. F. (2010). Linguistic alignment between people and computers. Journal of Pragmatics, 42, 2355-2368.

Brennan, S. E., \& Clark, H. H. (1996). Conceptual pacts and lexical choice in conversation. Journal of Experimental Psychology: Learning, Memory, and Cognition, 22, 1482-1493.

Breslow, N. E., \& Clayton, D. G. (1993). Approximate inference in generalized linear mixed models. Journal of the American Statistical Society, 88, 9-25.

Bybee, J. L. (1985). Morphology: A study of the relation between meaning and form. Philadelphia. PA: John Benjamins.

Bybee, J. L. (2010). Language, usage and cognition. Cambridge, England: Cambridge University Press.

Bybee, J. L., \& Slobin, D. I. (1982). Rules and schemas in the development and use of the English past tense. Language, 58, 265-289.

Chartrand, T. L., \& Bargh, J. A. (1999). The chameleon effect: The perception-behavior link and social interaction. Journal of Personality and Social Psychology, 76, 893-910.

Crepaldi, D., Rastle, K., Coltheart, M., \& Nickels, L. (2010). Fell primes fall, but does bell prime ball? Masked priming with irregularly-inflected primes. Journal of Memory and Language, 63, 83-99.

Delvaux, V., \& Soquet, A. (2007). The influence of ambient speech on adult speech productions through unintentional imitation. Phonetica, 64, 145-173.

Estival, D. (1985). Syntactic priming of the passive in English. Text: Interdisciplinary Journal for the Study of Discourse, 5, 7-22. 
Gallois, C., Ogay, T., \& Giles, H. (2005). Communication Accommodation Theory: A look back and a look ahead. In W. B. Gudykunst (Ed.), Theorizing about intercultural communication (pp. 121-148). Thousand Oaks, CA: Sage.

Garrod, S., \& Pickering, M. J. (2004). Why is conversation so easy? Trends in Cognitive Sciences, 8, 8-11.

Giles, H., Coupland, N., \& Coupland, J. (1991). Accommodation theory: Communication, context, and consequence. In H. Giles, J. Coupland, \& N. Coupland (Eds.), Contexts of accommodation: Developments in applied sociolinguistics (pp. 1-68). New York, NY: Cambridge University Press.

Giles, H., Taylor, D. M., \& Bourhis, R. (1973). Towards a theory of interpersonal accommodation through language: Some Canadian data. Language in Society, 2, 177-192.

Goldinger, S. D. (1998). Echoes of echoes? An episodic theory of lexical access. Psychological Review, 105, 251-279.

Gregory, S. W., Jr., \& Webster, S. (1996). A nonverbal signal in voices of interview partners effectively predicts communication accommodation and social status perceptions. Journal of Personality and Social Psychology, 70, 1231-1240.

Gries, S. T. (2005). Syntactic priming: A corpus-based approach. Journal of Psycholinguistic Research, 34, 365-399.

Haber, L. R. (1976). Leaped and leapt: A theoretical account of linguistic variation. Foundations of Language, 14, 211-38.

Hall, M. L., Ferreira, V. S., \& Mayberry, R. I. (2015). Syntactic priming in American Sign Language. PLoS One, 10, e0119611. doi:10.1371/journal.pone.0119611

Hay, J., Warren, P., \& Drager, K. (2006). Factors influencing speech perception in the context of a merger-in-progress. Journal of Phonetics, 34, 458-484.

Hruschka, D. J., Christiansen, M. H., Blythe, R. A., Croft, W., Heggarty, P., Mufwene, S. S., . . Poplack, S. (2009). Building social cognitive models of language change. Trends in Cognitive Sciences, 13, 464-469.

Jaeger, T. F. (2008). Categorical data analysis: Away from ANOVAs (transformation or not) and towards logit mixed models. Journal of Memory and Language, 59, 434-446.

Kundu, P., \& Cummins, D. D. (2013). Morality and conformity: The Asch paradigm applied to moral decisions. Social Influence, 8, 268-279.

Labov, W. (2007). Transmission and diffusion. Language, 83, 344-387.

McFarland, D. H. (2001). Respiratory markers of conversational interaction. Journal of Speech, Language, and Hearing Research, 44, 128-143.

Moder, C. L. (1992). Productivity and categorization in morphological classes (Unpublished doctoral dissertation). State University of New York at Buffalo.

Nass, C. I., Moon, Y., \& Carney, P. (1999). Are people polite to computers? Responses to computer-based interviewing systems. Journal of Applied Social Psychology, 29, 1093-1110.

Nass, C., Steuer, J., \& Tauber, E. R. (1994). Computers are social actors. In Proceedings of the SIGCHI Conference on Human Factors in Computing Systems (pp. 72-78). Boston, MA: ACM Press.

Natale, M. (1975). Convergence of mean vocal intensity in dyadic communication as a function of social desirability. Journal of Personality and Social Psychology, 32, 790-804.

Oviatt, S., Darves, C., \& Coulston, R. (2004). Toward adaptive conversational interfaces: Modeling speech convergence with animated personas. ACM Transactions on ComputerHuman Interaction, 11, 300-328.

Pardo, J. S. (2013). Measuring phonetic convergence in speech production. Frontiers in Psychology, 4, 1-5. 
Pardo, J. S., Gibbons, R., Suppes, A., \& Krauss, R. M. (2012). Phonetic convergence in college roommates. Journal of Phonetics, 40, 190-197.

Pickering, M. J., \& Garrod, S. (2004). Toward a mechanistic psychology of dialogue. Behavioral and Brain Sciences, 27, 169-190.

Pickering, M. J., \& Garrod, S. (2006). Alignment as the basis for successful communication. Research on Language and Computation, 4, 203-228.

Rácz, P., Beckner, C., Hay, J. B., \& Pierrehumbert, J. B. (2014). Rules, analogy, and social factors codetermine past-tense formation patterns in English. In Proceedings of the 2014 Joint Meeting of SIGMORPHON and SIGFSM (pp. 55-63). Baltimore $\mathrm{MD}$ : ACL.

Reeves, B., \& Nass, C. (1996). The media equation: How people treat computers, television, and new media like real people and places. Cambridge, England: Cambridge University Press.

Sancier, M. L., \& Fowler, C. A. (1997). Gestural drift in a bilingual speaker of Brazilian Portuguese and English. Journal of Phonetics, 25, 421-436.

Sherif, M. (1935). A study of some social factors in perception. Archives of Psychology, 27, 1 60

Soliz, J., \& Giles, H. (2014). Relational and identity processes in communication: A contextual and meta-analytical review of communication accommodation theory. In E. Cohen (Ed.), Communication yearbook 38 (pp. 106-143). Thousand Oaks, CA: Sage.

Stemberger, J. P., \& MacWhinney, B. (1986). Form-oriented inflectional errors in language processing. Cognitive Psychology, 18, 329-354.

Szmrecsanyi, B. (2005). Language users as creatures of habit: A corpus-based analysis of persistence in spoken English. Corpus Linguistics and Linguistic Theory, 1, 113-150.

Szmrecsanyi, B. (2006). Morphosyntactic persistence in spoken English: A corpus study at the intersection of variationist sociolinguistics, psycholinguistics, and discourse analysis. Berlin, Germany: Mouton de Gruyter.

Toma, C. L. (2014). Towards conceptual convergence: An examination of interpersonal adaptation. Communication Quarterly, 62, 155-178.

Veríssimo, J., \& Clahsen, H. (2009). Morphological priming by itself: A study of Portuguese conjugations. Cognition, 112, 187-194.

Webb, J. T. (1972). Interview synchrony: An investigation of two speech rate measures in an automated standardized interview. In A. W. Siegman \& B. Pope (Eds.), Studies in dyadic communication (pp. 55-70). Oxford, England: Pergamon Press.

\section{Author Biographies}

Clay Beckner is a postdoctoral research fellow at the New Zealand Institute of Language, Brain and Behaviour (NZILBB), working on the Wordovators project. The project is aimed at gaining a better understanding of how words are created and used, in a close collaboration between Northwestern University and NZILBB. His research interests include usage-based linguistics, sociolinguistics, morphosyntax, and the mental lexicon.

Péter Rácz is a postdoctoral research fellow at the New Zealand Institute of Language, Brain and Behaviour, working on the Wordovators project. He is very excited about language modeling and socially motivated linguistic variation.

Jennifer Hay is a professor in linguistics at the University of Canterbury and the founder and director of the New Zealand Institute of Language, Brain and Behaviour. She has published 
articles on morphology, laboratory phonology, and sociophonetics. She is one of the principal investigators of the Wordovators project.

Jürgen Brandstetter (MSc, Vienna Institute of Technology) is a doctoral student in humanrobot interaction at HITLabNZ, University of Canterbury. He studies the behavioral effects that groups of robots can have on humans.

Christoph Bartneck is an associate professor and director of postgraduate studies at HITLabNZ, University of Canterbury. He has a background in industrial design and humancomputer interaction. His research interests lie in the fields of social robotics, design science, and multimedia applications. 